\title{
Challenges of Glaucoma Management in Patients with Type I Boston Keratoprosthesis
}

\author{
Sara M AlHilali $\mathbb{D}$, Samar A AI-Swailem (D) \\ Cornea, External Eye Diseases and Refractive Surgery Division, King Khaled Eye Specialist Hospital, Riyadh, Saudi Arabia \\ Correspondence: Sara M AlHilali, Tel +00966II482I234 ext; 237I, Fax +4821234XI250, Email shilali@kkesh.med.sa
}

\begin{abstract}
Type I Boston keratoprosthesis is implanted in patients with severely diseased eyes who are considered poor candidates for traditional keratoplasty. Glaucoma is considered a major visual comorbidity following the implantation of type I Boston keratoprosthesis (KPro). Eyes that receive a Boston KPro are at high risk of progression of pre-existing glaucoma and the development of de novo glaucoma. Both complications can limit best-corrected visual acuity postoperatively. Diagnosis and surveillance for glaucoma in KPro eyes are fundamental to mitigate the risk of visual morbidity. However, managing these patients presents several challenges. The diagnosis of glaucoma after KPro implantation is usually hindered by inaccurate intraocular pressure (IOP) measurements and unreliable ophthalmic investigations such as visual field testing and optical coherence tomography (OCT) of the retinal nerve fiber layer (RNFL). In these eyes, medical management of glaucoma with topical medications is usually insufficient, and glaucoma surgery is usually warranted either prior to or during KPro implantation. Options for glaucoma surgery include glaucoma drainage device (GDD) and cyclodestructive procedures. The aim of this article is to highlight the incidence, pathophysiology, diagnosis, and management options of glaucoma in eyes that have undergone type I Boston keratoprosthesis.
\end{abstract}

Keywords: glaucoma, Boston keratoprosthesis, management, surveillance, complications

\section{Introduction}

Glaucoma is considered a major visual comorbidity following the implantation of type I Boston Keratoprosthesis (KPro). ${ }^{1-4}$ Previous literature indicates poor postoperative visual acuity in eyes with pre-existing glaucoma that undergo KPro implantation. The poor visual outcomes are due to the rapid progression of glaucoma and optic nerve damage, in addition to the difficulty in reliably assessing intraocular pressure and visual fields. ${ }^{5,6}$ Approximately $50 \%$ of eyes undergoing KPro implantation develop glaucoma due to various etiologies, leading to further visual decline if not diagnosed and managed in a timely manner. ${ }^{5}$ Thus, monitoring and controlling intraocular pressure in patients with Type I Boston Keratoprosthesis play a major role in visual outcomes.

The aim of this article is to highlight the incidence, pathophysiology, diagnosis, and management of glaucoma in eyes that have underwent type I Boston Keratoprosthesis. Articles between 1990 and 2021 on glaucoma, surveillance, and management in Boston KPro patients were identified in PubMed and reviewed.

\section{Incidence of Glaucoma in Eyes with Type I Boston Keratoprosthesis}

There are multiple studies reporting the incidence of pre-existing glaucoma in eyes scheduled to receive the KPro, and the incidence of new-onset glaucoma following KPro implantation. Lenis et al reported that 98 of 137 eyes (71.5\%) had glaucoma prior to KPro implantation, and, of the remaining 39 eyes, 5 eyes (12.8\%) developed glaucoma requiring medical or surgical intervention. ${ }^{2}$ Lee et al reported that 35 of 48 eyes (72.9\%) had preexisting glaucoma and 4 of 48 eyes (8.3\%) developed de novo glaucoma following keratoprosthesis implantation. ${ }^{7}$ A report in 2013 concluded that $67 \%$ of eyes were diagnosed with glaucoma prior to KPro implantation and 50\% developed glaucoma postoperatively. ${ }^{1} \mathrm{~A}$ study from the University of California of 30 eyes demonstrated that $60 \%$ had preoperative glaucoma and $27 \%$ of eyes developed new-onset glaucoma postoperatively. ${ }^{4}$ In 2008, a study of 18 eyes found that 11 eyes (61\%) had glaucoma 
prior to keratoprosthesis surgery, and 7 eyes (39\%) developed glaucoma postoperatively. ${ }^{8}$ In summary, the prevalence of pre-existing glaucoma in eyes scheduled for KPro implantation ranges from $61 \%$ to $72.9 \%$, while the incidence of de novo glaucoma ranges from $8 \%$ to $50 \%$.

\section{Etiology of Glaucoma Following KPro Implantation}

The pathophysiology of the development of glaucoma following Keratoprosthesis implantation is multifactorial. ${ }^{9}$ Some authors postulate that secondary angle closure develops due to progressive peripheral anterior synechia postoperatively. ${ }^{1}$ Postoperative synechia have been reported in imaging studies using anterior segment optical coherence tomography (ASOCT). ${ }^{10-13}$ Kang et al published a paper in 2013 investigating changes in anterior chamber depth and angle in eyes that had undergone KPro implantation. ${ }^{10}$ They used serial AS-OCT and found that at a mean of 13 months postoperatively, anterior chamber depth decreased by $1.6 \mathrm{~mm}$, and that $73 \%$ of eyes had peripheral anterior synechia or approximately 7 clock hours of iris-back plate touch. ${ }^{10}$ In a subsequent paper, Kang et al reviewed the AS-OCT of 52 eyes that received the type 1 Boston KPro and concluded that $86.5 \%$ of eyes had peripheral anterior synechia. ${ }^{11}$ Many have advocated that a pediatric sized back plate $(7 \mathrm{~mm})$ should be used to reduce shallowing of the anterior chamber and the contact of the iris to the back plate. However, Kang et al's outcome show similar rates of peripheral anterior synechia (PAS) formation in eyes that received $8.5 \mathrm{~mm}$ versus $7 \mathrm{~mm}$ sized back plates. ${ }^{11}$ Nevertheless, surgeons do frequently perform total iridectomy along with KPro implantation, yet it fails to eliminate the risk of glaucoma. Dohlman reported that 21 out of 36 eyes did develop glaucoma despite total iridectomy. ${ }^{9}$ Some have hypothesized an inflammatory component to glaucoma in KPro eyes. Notably, a paper in 2021 compared tear film cytokines of KPro patients in eyes with and without glaucoma and found significantly elevated levels of four cytokines (TNF-a, IL-1 $\beta$, FGF-basic, IFN- $\gamma$ ) in KPro eyes with glaucoma. ${ }^{14}$ Interestingly, a case-controlled study by Geoffrion and Harissi-Dagher which enrolled 118 eyes found that a preoperative diagnosis of autoimmune or ocular surface diseases precipitated glaucoma progression, while a diagnosis of stromal or endothelial disorders was protective against glaucoma progression in KPro eyes postoperatively. ${ }^{15}$ They proposed that the stromal and endothelial disorders are limited to the corneal layers with preserved angle anatomy and did not precipitate anterior segment inflammation. ${ }^{15}$

\section{Glaucoma Surveillance Post KPro}

Eyes that have undergone KPro implantation are at a significantly elevated risk of developing glaucoma, which is considered a major debilitating disease. Therefore, regular monitoring for the development of glaucoma and progression is critical. This includes, documenting baseline IOP, optic nerve assessment by direct visualization or optical coherence tomography (OCT) and visual field testing.

\section{IOP Measurement and Assessment of Glaucoma Following KPro Implantation}

Measurement of intraocular pressure (IOP) in KPro eyes is challenging. KPro implantation changes the biomechanics of the scleral wall, resulting in inaccurate IOP measurements. At one time, tactile tension was considered the most practical method for assessing post-keratoprosthesis IOP. ${ }^{9,16}$ However, the lack of repeatability among examiners makes it inadequate. Some studies have compared the variability in IOP estimation using different methods in KPro eyes. ${ }^{17-20}$ A group of investigators compared IOP measurements using the Tono-pen versus Schiotz tonometry in 5 donor eyes, where a surgeon sutured a keratoprosthesis to the central cornea and an infusion line was connected and set at 10, 20, 30 and $40 \mathrm{mmHg} .{ }^{18}$ The authors concluded that Schiotz Tonometry had a lower median absolute error compared to the Tono-pen at both the temporal sclera $(5.4 \mathrm{vs} 39 \mathrm{mmHg}, P<0.001)$ and the corneoscleral limbus $(4.9 \mathrm{vs} 15 \mathrm{mmHg}, P=$ 0.03). ${ }^{18}$ Lin et al investigated the accuracy of scleral IOP measurement using pneumotonometry in 6 cadaver eyes with Boston KPro, cannulated with an infusion line. ${ }^{17}$ They found that scleral IOP measured higher than corneal IOP by a mean of $13 \mathrm{mmHg}$, and there was a positive linear correlation with the actual IOP and scleral IOP measurement postKPro implantation. ${ }^{17}$ Lin et al also confirmed that the inferotemporal quadrant is the most accurate location for measuring IOP. ${ }^{17}$ Another study compared digital palpation, trans-scleral pneumotonometry and trans-palpebral (over the upper eyelid) Diaton tonometry. ${ }^{19}$ This study found that the Diaton tonometer was similar to digital palpation for estimating IOP, while trans-scleral pneumotononmetry yielded higher values than anticipated. ${ }^{19}$ The literature has documented multiple attempts at developing innovative designs to create an implantable intraocular transducer to 
continuously measure intraocular pressure variations throughout the day. ${ }^{21,22} \mathrm{~A}$ group from Germany has successfully developed an electromagnetic device by the name "Eyemate-IO" (Implandata, Hannover, Germany) which is a sensor ring that is implanted in the ciliary sulcus and enables continuous telemetric IOP monitoring and transfers data to a handheld device. ${ }^{23}$ A study published in the American Journal of Ophthalmology reported one-year outcomes of 12 eyes that had undergone Eyemate-IO implantation along with Type 1 Boston Keratoprosthesis implantation. ${ }^{24}$ The results of the telemetric system were compared to IOP assessment by digital palpation by two senior ophthalmologists and the outcomes were promising for monitoring IOP in KPro eyes postoperatively. ${ }^{24}$ However, further studies with a larger sample size and longer follow-up duration are needed prior to establishing the Eyemate-IO as a substitute for other methods for measuring IOP. ${ }^{24}$ In summary, digital palpation remains the recommended method for monitoring IOP in KPro patients, given that it is performed by an experienced ophthalmologist. ${ }^{25}$ The Tono-pen and trans-scleral pneumatonometer are considered the least accurate methods for measuring IOP in KPro patients ${ }^{25}$ [Table 1].

\section{Optic Nerve Assessment and Visual Field Testing}

Optic disc evaluation at baseline and serial follow-ups is crucial, either by direct visualization with slit-lamp examination or by documenting the cup-to-disc ratio with photography. Evaluation of retinal nerve fiber layer (RNFL) at the optic nerve head with OCT is possible and could be a useful tool for monitoring glaucoma progression in KPro eyes. ${ }^{26,27}$ Retinal nerve fiber layer (RNFL) thinning is the most common indicator of glaucoma progression. In fact, RNFL thinning may precede defects in the visual field. ${ }^{28}$ Therefore, serial assessment of RNFL in KPro eyes could potentially aid in diagnosing glaucoma progression. However, Khoueir et al reported a high rate of segmentation error of the retinal nerve fiber layer (RNFL) in spectral domain OCT (SD-OCT) and attributed it the decreased reflectivity of RNFL in KPro eyes with advanced glaucoma. ${ }^{29}$ Thus, better OCT testing parameters are needed for KPro patients. ${ }^{29}$

Visual field testing can also be used in the assessment of functional glaucomatous changes in KPro eyes. ${ }^{26,27}$ Normally, Goldmann visual field (GVF) test is most commonly used to delineate visual field defects in KPro eyes. A novel method used digital planimetric analysis of GVFs was proposed by Ali et al. ${ }^{27}$ This method involved using the V4e isopter beyond the central 24 degrees for all patients, and a loss of more than $30 \%$ of surface area was considered as visual field deterioration in KPro eyes ${ }^{27}$ A cross-sectional study that included 6 eyes that received the KPro device with no previous history of glaucoma, concluded that Goldmann manual perimetry was a reproducible test will all eyes showing good reliability indices. ${ }^{30}$ Akpek et al tested the reliability of different glaucoma investigations in patients after Boston type 1 KPro implantation versus eyes with penetrating keratoplasty as controls. ${ }^{31}$ They concluded that the Humphrey 10-2 visual field test and RNFL thickness with the OCT are both repeatable and reliable for detecting glaucomatous progression in KPro eyes. ${ }^{31}$

\section{Management of Glaucoma Following KPro Implantation \\ Medical}

Topical antiglaucoma medications can be used to control intraocular pressure. Aqueous suppressants and medications that increase the uveoscleral outflow are preferred, because drops affecting the trabecular outflow have little to no value

Table I Accuracy of Different Methods of Intraocular Pressure Measurement in KPro Eyes

\begin{tabular}{|l|l|l|l|}
\hline Study & Study Method & $\begin{array}{l}\text { N. of } \\
\text { Eyes }\end{array}$ & Result \\
\hline $\begin{array}{l}\text { Lin et al } \\
(2014)^{17}\end{array}$ & Pneumotonometer & 6 & Scleral IOP measured higher than corneal IOP by a mean of I3mmHg \\
\hline $\begin{array}{l}\text { Estrovich } \\
\text { et al } \\
(2015)^{18}\end{array}$ & $\begin{array}{l}\text { Schiotz tonometer vs Handheld tonometer } \\
\text { (Tono-pen) }\end{array}$ & 5 & $\begin{array}{l}\text { Schiotz Tonometry had a lower median absolute error compared to } \\
\text { Tono-pen at both temporal sclera }(5.4 \text { vs } 39 \mathrm{mmHg} \text { P<0.00I) and } \\
\text { corneoscleral limbus (4.9 vs I5mmHg, P }=0.03)\end{array}$ \\
\hline $\begin{array}{l}\text { Wilensky } \\
\text { et al } \\
(2013)^{19}\end{array}$ & $\begin{array}{l}\text { Digital palpation vs Trans-scleral } \\
\text { pneumotonometer vs Trans-palpebral Diaton } \\
\text { tonometer }\end{array}$ & $\begin{array}{l}\text { Digital IOP estimation was similar to Diaton tonometer } \\
\text { Trans-scleral pneumotonometer consistently yielded higher IOP values, } \\
\text { compared to Diaton tonometer }\end{array}$ \\
\hline
\end{tabular}


due to the extensive angle closure in most KPro eyes. ${ }^{12}$ The general recommendation is to also avoid prostaglandin analogues as they can exacerbate preexisting macular edema that is common in these eyes. ${ }^{32}$ Furthermore, to minimize compromising the ocular surface, it the use preservative-free agents are recommended. Oral carbonic anhydrase inhibitors are an alternative if the patient has normal kidney function and is monitored regularly for side effects.

\section{Surgical}

In KPro eyes there is no consensus on the ideal type and timing of surgical intervention for glaucoma. Regardless, there is a trend toward the implantation of glaucoma drainage devices (GDDs) either prior to or concurrently with the type I Boston KPro.,33-36 Tube placement in these eyes is generally performed at the pars plana following adequate pars plana vitrectomy ${ }^{34,37}$ as the anterior chamber is not an ideal location due to crowding of angle structures and the higher risk of tube occlusion by the vitreous or iris. ${ }^{1,38}$ A posteriorly positioned tube is preferable as it eases the fitting of a bandage contact lens (BCL). ${ }^{37}$ Alternately, there are multiple reports about BCL causing conjunctival erosion and leading to tube exposure, ${ }^{35,38}$ Patel reviewed the charts of 130 eyes with both KPro and a GDD and found that 19 eyes developed tube erosions, 7 of these 19 eyes resolved completely after changing the diameter of the contact lens. ${ }^{39}$

The Ahmed glaucoma drainage device implant (AGI) and Baerveldt glaucoma drainage device implant (BGI) are the two most frequently used GDDs. However, there is limited data in the literature comparing the two devices. Huh et al reported the results of 12 eyes that received AGI and 5 eyes that received BGI and concluded that posteriorly positioned pars plana GDD has a low rate of postoperative complications. ${ }^{34}$ However, they did not include the differences in outcomes between the two devices. ${ }^{34}$

Cyclodestructive procedures such as trans-scleral cyclophotocoagulation (TSCPC) can be used to treat refractory glaucoma with or without prior GDD implantation. ${ }^{8,40}$ Rivier et al reported the results of 18 eyes that had undergone TSCPC. $^{8}$ Their findings indicate that mean postoperative IOP significantly decreased at last follow-up, and only 2 eyes developed postoperative complications including conjunctival dehiscence and fungal endophthalmitis. ${ }^{8}$ Jabbour et al had contradictory findings in a retrospective case series of 18 eyes that had undergone $\mathrm{CPC} .^{40}$ They found that glaucoma progressed despite CPC in 7 eyes had and 3 eyes that had well-controlled IOP initially, failed to respond later, and 2 eyes developed complications including cystoid macular edema and bacterial endophthalmitis. ${ }^{40}$ Endocyclophotocoagulation (ECP) has been advocated as another treatment modality in these eyes, ${ }^{41}$ as it has the advantage of avoiding conjunctiva trauma and no tube-related complications. However, there is limited data on the long-term outcomes and complications of ECP in KPro eyes.

Currently there is no standard on the timing of glaucoma surgery in KPro implantation. Some studies evaluated the outcomes and safety of prior or concurrent GDD and KPro implantation, ${ }^{2,3,42,43}$ and found that eyes with early or concurrent glaucoma surgical intervention did retain vision better compared to patients with late or no intervention. Additionally, concurrent glaucoma surgery did not increase the rate of postoperative complications compared to KPro implantation alone. Geoffrion et al retrospectively reviewed 100 eyes of patients who had undergone type 1 Boston KPro and found that all eyes with preexisting glaucoma without any prior or concurrent surgical intervention for glaucoma had progressive worsening of glaucoma, despite controlled IOP preoperatively. ${ }^{43}$ Therefore, it is imperative to ensure proper IOP control by implanting a GDD device either prior to or concurrently with KPro implantation, especially in patients with preoperative glaucoma. The exact timing of pre-KPro glaucoma surgery is not clearly described in the existing literature although some have advocated implanting a GDD device as soon as 6 weeks prior to KPro surgery. ${ }^{2}$

\section{Conclusion}

This review presents the multiple challenges in the diagnosis and monitoring of glaucoma in eyes with type I Boston keratoprosthesis. To assess progression of glaucoma in these patients we recommend careful preoperative and postoperative assessment of intraocular pressure and serial postoperative disc photos to monitor cup to disc ratio and visual field testing. Surgical options are limited to glaucoma drainage devices and cyclodestructive procedures, careful planning of the type and timing of the glaucoma surgery is crucial. Data from the studies included in this review help to navigate the ophthalmologists in managing patients who have received KPro. 


\section{Disclosure}

The authors report no conflicts of interest in this work.

\section{References}

1. Kamyar R, Weizer JS, De paula FH, et al. Glaucoma associated with Boston type I keratoprosthesis. Cornea. 2012;31(2):134-139. doi:10.1097/ ICO.0b013e31820f7a32

2. Lenis TL, Chiu SY, Law SK, Yu F, Aldave AJ. Safety of concurrent Boston type I keratoprosthesis and glaucoma drainage device implantation. Ophthalmology. 2017;124(1):12-19. doi:10.1016/j.ophtha.2016.08.003

3. Crnej A, Paschalis EI, Salvador-Culla B, et al. Glaucoma progression and role of glaucoma surgery in patients with Boston keratoprosthesis. Cornea. 2014;33(4):349-354. doi:10.1097/ICO.0000000000000067

4. Bradley JC, Hernandez EG, Schwab IR, Mannis MJ. Boston type 1 keratoprosthesis: the University of California Davis experience. Cornea. 2009;28(3):321-327. doi:10.1097/ICO.0b013e31818b8bfa

5. Vajaranant TS, Liu J, Wilensky J, Cortina MS, Aref AA. Innovative approaches to glaucoma management of Boston keratoprosthesis type 1. Curr Ophthalmol Rep. 2016;4(3):147-153. doi:10.1007/s40135-016-0102-3

6. Srikumaran D, Munoz B, Aldave AJ, et al. Long-term outcomes of Boston type 1 keratoprosthesis implantation: a retrospective multicenter cohort. Ophthalmology. 2014;121(11):2159-2164. doi:10.1016/j.ophtha.2014.05.030

7. Lee R, Khoueir Z, Tsikata E, Chodosh J, Dohlman CH, Chen TC. Long-term visual outcomes and complications of Boston keratoprosthesis type II implantation. Ophthalmology. 2017;124(1):27-35. doi:10.1016/j.ophtha.2016.07.011

8. Rivier D, Paula JS, Kim E, Dohlman CH, Grosskreutz CL. Glaucoma and keratoprosthesis surgery. J Glaucoma. 2009;18(4):321-324. doi:10.1097/ IJG.0b013e3181815485

9. Netland PA, Terada H, Dohlman CH. Glaucoma associated with keratoprosthesis. Ophthalmology. 1998;105(4):751-757. doi:10.1016/S0161-6420 (98) $94034-9 \mathrm{q}$

10. Kang JJ, Allemann N, De La Cruz J, Cortina MS. Serial analysis of anterior chamber depth and angle status using anterior segment optical coherence tomography after Boston keratoprosthesis. Cornea. 2013;32(10):1369-1374. doi:10.1097/ICO.0b013e3182a0cff5

11. Kang JJ, Allemann N, Vajaranant T, de la Cruz J, Cortina MS. Anterior segment optical coherence tomography for the quantitative evaluation of the anterior segment following Boston keratoprosthesis. PLoS One. 2013;8(8). doi:10.1371/annotation/c3ad2f44-5b6f-430a-b5cb-993eae6644ad

12. Panarelli JF, Ko A, Sidoti PA, Garcia JP, Banitt MR. Angle closure after Boston keratoprosthesis. J Glaucoma. 2013;22(9):725-729. doi:10.1097/ IJG.0b013e318259b2fc

13. Qian CX, Hassanaly S, Harissi-Dagher M. Anterior segment optical coherence tomography in the long-term follow-up and detection of glaucoma in Boston type I keratoprosthesis. Ophthalmology. 2015;122(2):317-325. doi:10.1016/j.ophtha.2014.08.007

14. Geoffrion D, Robert MC, Di PA, Koenekoop RK, Agoumi Y, Harissi-Dagher M. Tear film cytokine profile of patients with the Boston keratoprosthesis type 1: comparing patients with and without glaucoma. Investig Ophthalmol Vis Sci. 2021;62(4):11-13.

15. Geoffrion D, Harissi-Dagher M. Glaucoma risk factors and outcomes following Boston keratoprosthesis type 1 surgery. Am J Ophthalmol. 2021;226:56-67. doi:10.1016/j.ajo.2021.01.006

16. Rubinfeld RS, Cohen EJ, Laibson PR, Arentsen JJ, Lugo MGG, Genvert GI. The accuracy of finger tension for estimating intraocular pressure after penetrating keratoplasty. Ophthalmic Surg Lasers. 1998;29(3):213-215. doi:10.3928/1542-8877-19980301-07

17. Lin CC, Chen A, Jeng BH, Porco TC, Ou Y, Han Y. Scleral intraocular pressure measurement in cadaver eyes pre- and postkeratoprosthesis implantation. Investig Ophthalmol Vis Sci. 2014;55(4):2244-2250. doi:10.1167/iovs.13-13153

18. Estrovich IE, Shen C, Chu Y, et al. Schiotz tonometry accurately measures intraocular pressure in Boston type 1 keratoprosthesis eyes. Cornea. 2015;34(6):682-685. doi:10.1097/ICO.0000000000000406

19. Liu J, Vajaranant T, Cortina M, Wilensky J. Agreement among transpalpebral, transcleral and tactile intraocular pressure measurements in eyes with type 1 Boston keratoprosthesis. Invest Ophthalmol Vis Sci. 2013;54:3472. doi:10.1167/iovs.12-11169

20. Ono T, Mori Y, Nejima R, et al. Short-term interim results of clinical outcomes and complications after implantation of Boston keratoprosthesis in Japanese patients. Cornea. 2020;39(Suppl 1):S28-S33. doi:10.1097/ICO.0000000000002453

21. Krug A, Kompa S, Schrage NF. The Aachen-keratoprosthesis - A flexible KPro that permits intraocular pressure measurement. Int J Artif Organs. 2002;25(3):238-242. doi:10.1177/039139880202500311

22. Marnat L, Ouda MH, Arsalan M, Salama K, Shamim A. On-chip implantable antennas for wireless power and data transfer in a glaucomamonitoring SoC. IEEE Antennas Wirel Propag Lett. 2012;11:1671-1674. doi:10.1109/LAWP.2013.2240253

23. Koutsonas A, Walter P, Roessler G, Plange N. Implantation of a novel telemetric intraocular pressure sensor in patients with glaucoma (ARGOS study): 1-year results. Invest Ophthalmol Vis Sci. 2015;56(2):1063-1069. doi:10.1167/iovs.14-14925

24. Enders P, Hall J, Bornhauser M, et al. Telemetric intraocular pressure monitoring after Boston keratoprosthesis surgery using the eyemate-IO sensor: dynamics in the first year. Am J Ophthalmol. 2019;206:256-263. doi:10.1016/j.ajo.2019.02.025

25. Melki S, Lopez M, Dohlman C. Boston keratoprosthesis update. Massachusetts Eye Ear Infirm; 2009.

26. Xing D, Chiou C, Mannis M, Keltner J. Visual fields and retinal nerve fiber layer thickness after Boston keratoprosthesis. Invest Ophthalmol Vis Sci. 2010;51(13):1142.

27. Ali MH, Dikopf MS, Finder AG, et al. Assessment of glaucomatous damage after Boston keratoprosthesis implantation based on digital planimetric quantification of visual fields and optic nerve head imaging. Cornea. 2018;37(5):602-608. doi:10.1097/ICO.0000000000001544

28. Quigley HA, Addicks EM, Green WR. Optic nerve damage in human glaucoma. III. Quantitative correlation of nerve fiber loss and visual field defect in glaucoma, ischemic neuropathy, papilledema, and toxic neuropathy. Arch Ophthalmol. 1982;100(1):135-146. doi:10.1001/ archopht.1982.01030030137016

29. Khoueir Z, Jassim F, Braaf B, et al. Three-dimensional optical coherence tomography imaging for glaucoma associated with Boston keratoprosthesis type I and II. J Glaucoma. 2019;28(8):718-726. doi:10.1097/IJG.0000000000001280

30. Quercia AZF, Silva LD, de Oliveira F, Teixeira SH, de Sousa LB, de Oliveira LA. Visual field characteristics of type I Boston keratoprosthesis patients without glaucoma. J Glaucoma. 2021;30:532-536. 
31. Akpek EK, Karakus S, Yohannan J, et al. Reliability of several glaucoma tests in patients with Boston type 1 keratoprosthesis. Cornea. 2021:1-7. doi:10.1097/ICO.0000000000002484

32. Patil AJ, Vajaranant TS, Edward DP. Bimatoprost - a review. Expert Opin Pharmacother. 2009;10:2759-2768. doi:10.1517/14656560903292649

33. Law SK, Huang JS, Nassiri N, et al. Technique of combined glaucoma tube shunt and keratoprosthesis implantation. J Glaucoma. 2014;23(8):501507. doi:10.1097/IJG.0b013e31829d9c00

34. Huh ES, Aref AA, Vajaranant TS, De La Cruz J, Chau FY, Cortina MS. Outcomes of pars plana glaucoma drainage implant in Boston type 1 keratoprosthesis surgery. J Glaucoma. 2014;23(1):e39-e44. doi:10.1097/IJG.0b013e31829e55f8

35. Li JY, Greiner MA, Brandt JD, Lim MC, Mannis MJ. Long-term complications associated with glaucoma drainage devices and Boston keratoprosthesis. Am J Ophthalmol. 2011;152(2):209-218. doi:10.1016/j.ajo.2011.01.034

36. Aldave AJ, Kamal KM, Vo RC, Yu F. The Boston type I keratoprosthesis. Improving outcomes and expanding indications. Ophthalmology. 2009;116(4):640-651.

37. Vajaranant TS, Blair MP, McMahon T, Wilensky JT, De La Cruz J. Special considerations for pars plana tube-shunt placement in Boston type 1 keratoprosthesis. Arch Ophthalmol. 2010;128(11):1480-1482.

38. Chew HF, Ayres BD, Hammersmith KM, et al. Boston keratoprosthesis outcomes and complications. Cornea. 2009;28(9):989-996. doi:10.1097/ ICO.0b013e3181a186dc

39. Patel S, Takusagawa H, Shen L, Dohlman C, Grosskreutz C. Long-term complications associated with glaucoma drainage devices and Boston keratoprosthesis. Am J Ophthalmol. 2012;154:207-208. doi:10.1016/j.ajo.2012.02.033

40. Jabbour S, Harissi-Dagher M, Agoumi Y, Singh H, Robert MC. Cyclophotocoagulation in the control of glaucoma in patients with the Boston keratoprosthesis type 1. Cornea. 2020;39(2):181-185. doi:10.1097/ICO.00000000000002064

41. Talajic JC, Agoumi Y, Gagn S, Moussally K, Harissi-Dagher M. Prevalence, progression, and impact of glaucoma on vision after Boston type 1 keratoprosthesis surgery. Am J Ophthalmol. 2012;153(2):267-274.e1. doi:10.1016/j.ajo.2011.07.022

42. Lin M, Bhatt A, Haider A, et al. Vision retention in early versus delayed glaucoma surgical intervention in patients with Boston Keratoprosthesis type 1. PLoS One. 2017;12(8):1-9.

43. Geoffrion D, Hassanaly SI, Marchand M, Daoud R, Agoumi Y, Harissi-Dagher M. Assessment of the role and timing of glaucoma surgery in Boston keratoprosthesis type 1 patients. Am J Ophthalmol. 2022;235:249-257. doi:10.1016/j.ajo.2021.09.005

Clinical Ophthalmology

\section{Publish your work in this journal}

Clinical Ophthalmology is an international, peer-reviewed journal covering all subspecialties within ophthalmology. Key topics include: Optometry; Visual science; Pharmacology and drug therapy in eye diseases; Basic Sciences; Primary and Secondary eye care; Patient Safety and Quality of Care Improvements. This journal is indexed on PubMed Central and CAS, and is the official journal of The Society of Clinical Ophthalmology (SCO). The manuscript management system is completely online and includes a very quick and fair peer-review system, which is all easy to use. Visit http://www. dovepress.com/testimonials.php to read real quotes from published authors.

Submit your manuscript here: https://www.dovepress.com/clinical-ophthalmology-journal 